\title{
Analysis of Hybrid Relaying in Cooperative WLAN
}

\author{
Tauseef Jamal $^{1}$, Paulo Mendes ${ }^{1}$, André Zúquete ${ }^{2}$ \\ ${ }^{1}$ SITILabs, University Lusofona, Campo Grande 376, 1749-024 Lisbon, Portugal \\ \{tauseef.jamal, paulo.mendes\}@ulusofona.pt \\ ${ }^{2}$ DETI, IEETA, University of Aveiro Campus Universitário de Santiago 3810-193 Aveiro, Portugal \\ andre.zuquete@ua.pt
}

\begin{abstract}
An ever-growing demand for higher data-rates has facilitated the growth of wireless networks in the past decades. Nevertheless, wireless technologies face performance limitations due to unstable wireless conditions and mobility of devices. In face of multi-path propagation and low data-rate stations, cooperative relaying promises gains in performance and reliability. However, cooperation procedures are unstable and introduce overhead that can endanger performance. In this paper we analyze the performance of a hybrid relaying protocol build based on the combination of opportunistic and broadcast-based relaying approaches. Hybrid relaying aims to increase the transmission capacity of wireless networks (proactive operation) when compared to proactive opportunistic and broadcast-based approaches due to rectifying the setbacks involved in those approaches, while adding a reactive approach to recover from failed transmissions.
\end{abstract}

\section{INTRODUCTION}

Cooperative communications that take advantage of the broadcasting nature of wireless environments have shown excellent performance in both theoretical analysis and implementations [1]. Cooperation occurs when overhearing relays assist the communication between source to destination, by transmitting different copies of the same signal from different locations, generating spatial diversity that allows the destination to get independently faded versions of the signal. Such copies can be combined at the destination to obtain an error-free signal [2]. Cooperative communication at the physical layer requires extra computation and synchronization capabilities. However, the cooperation at the Medium Access Control (MAC) level can provide performance gains [3], [4], even without the aforementioned capabilities. Cooperation at MAC layer is expected to provide answers to: when to use cooperation, whom to cooperate with, and how to cooperate.

In our previous work we proposed a cooperative relaying framework, RelaySpot [3], [5], which considers cooperation at MAC layer to answer the above mentioned questions. RelaySpot defines three basic components: opportunistic relay selection (proactive opportunistic behavior), cooperative relay scheduling (proactive broadcast behavior) and relay switching (reactive behavior). The proposal for opportunistic relay selection has been presented in [6], [7]. In this paper we show how a hybrid relaying approach such as RelaySpot, can perform better than opportunistic and broadcast-based relaying approaches.

RelaySpot uses a hybrid relaying approach aiming to mitigate the problems posed by fading and by the presence of low data-rate nodes. With RelaySpot, relays are self-elected if within a cooperation area defined for a source-destination pair, and after overhearing a good frame from the source. Moreover, Access Points (APs) or destination nodes are able to select the best set of relays based on the information provided by them during a predefined reception window. In this paper we consider a diversity of one during the experimental evaluation, which means that AP select only one relay. Simulation results show that with our hybrid protocol, standard 802.11 networks are able to offer ubiquitous high data-rate coverage and throughput, with reduced latencies.

The paper is organized as follows. Section II presents the related work. In section III we provide a description of the operation of hybrid cooperative relaying approaches. While section IV presents the performance evaluation of RelaySpot, an example of a hybrid cooperative relaying solution. Section $\mathrm{V}$ presents a summary of our findings and our conclusions.

\section{RELATED WORK}

Relaying at MAC layer consists of two phases: relay selection phase and cooperation phase. In first phase a relay is selected and then in the second phase transmission via selected relay takes place. Relaying protocols at MAC layer can either be proactive or reactive, as shown in Figure 1. In proactive relaying the source, destination or potential relay replaces the slow direct communication with a fast dual-hop relayed communication, aiming to improve the data-rate. In case of reactive relaying, relays forward data to the destination when the direct communication fails, avoiding retransmissions.

Common examples of proactive source-based cooperative relaying schemes at the MAC layer use one relay [8] or two relays in parallel [9]. Source-based relaying approaches such as CoopMAC [8] require the sources to maintain a table of Channel State Information (CSI) that is updated by potential relays based upon periodic broadcasts. One example of proactive destination-based relaying schemes at the MAC layer is a relay enabled DCF (rDCF) protocol [10]. In rDCF relays maintain a willingness list that contains the IDs of the source-destination pairs that a relay can help. Periodically, each potential relay broadcast its willingness list. In general such approaches undergo two main problems: channel estimation and periodic broadcasts, which introduce overhead that is problematic in mobile scenarios. In case of FairMAC [11] each relay node maintains an additional infinite queue to store the frames to be relayed.

While the mentioned proactive approaches rely upon broadcast, Opportunistic Relay Protocol (ORP) [12] does not. 


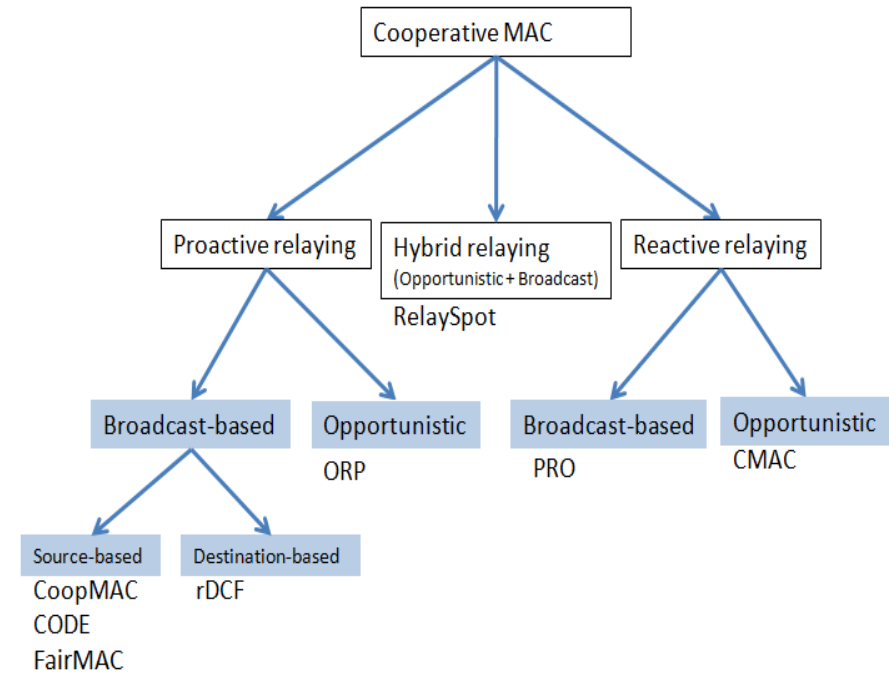

Figure 1. Cooperative MAC Taxonomy.

ORP does not rely on CSI for relay selection: The source opportunistically makes a frame available for relaying and all potential relays try to forward the frame within the time constraint. However, relays back-off every time they forward. Another drawback of this approach is that the source does not know the availability of a relay, and therefore, it does not know the rate of the source-relay and relay-destination channels.

Reactive cooperative methods such as PRO [13] rely on relays to decide to re-transmit on behalf of the source when the direct transmission fails. With PRO [13], relays are selected among a set of overhearing nodes in two phases: First, a local qualification process takes place at potential relays, leading to the identification of qualified relays. In a second phase, qualification information is broadcasted, allowing qualified relays to set scheduling priorities. In Cooperative Communication MAC (CMAC) [14] each node stores the source node data frame. If no ACK is overheard the relay opportunistically forwards the stored data frame on behalf of source.

In general, both proactive broadcast-based and opportunistic approaches have their pros and cons, depending on individual protocols. For example, none of the protocols address the relay failure issues or compensation of poor relay selection. Hybrid approaches on the other hand combine both proactive opportunistic and broadcast approaches: relays are elected opportunistically based on local parameters, while the sources or destinations try to organized the relays by selecting one over several potential candidates.

Most of the prior art consider proactive relaying due to its advantage of replacing poor links, while reactive approaches have less impact because the relaying occurs only for failed data frame. Hybrid approaches combines reactive and proactive approaches, by reacting to failed communications (direct link or relay transmission).

In summary hybrid approaches are promising because: they are expected to react fast to channel variations due to local election of relays (opportunistic behavior); they can rectify poor relay selections (broadcast-based behavior); and are able to react to direct as well as relay link failures (reactive behav- ior). Broadcast-based approaches select relays cooperatively in a centralized manner, but for this they construct a global map of neighborhood which is a drawback. Opportunistic approaches select a relay in a distributed manner with no additional overhead, but have the risk of collisions, failed attempts and poor selections. Hybrid approaches can bring broadcast-based behavior into opportunistic one without usage of additional overhead and maintenance of global map. As a result relays are selected with minimum coordination and the risk of poor opportunistic relay selection can be rectified.

However, it is still not clear the advantages that hybrid relaying has in relation to proactive broadcast-based and opportunistic in term of overall network capacity in scenarios with varying density. Hence, this paper aims to investigate if the combination of both types of (proactive broadcast-based and opportunistic) relaying is better or not, by comparing RelaySpot with broadcast-based and opportunistic mechanisms.

\section{Hybrid CoOperative RELAYING}

As mentioned before, RelaySpot is an example of a hybrid cooperative relaying protocol: It applies a proactive opportunistic approach to react fast to channel variations based on a local election of relays; a broadcast approach to rectify poor relay selections; and a reactive approach able to react to direct as well as relay link failures. This section presents the RelaySpot protocol in term of its proactive and reactive operations.

\section{A. Proactive Operation}

Figure 2, illustrates the RelaySpot operation in a scenario with a poor direct link between source and destination.

In this scenario, when the destination observes poor datarate, it implicitly asks for relaying by sending a cooperative Clear To Send (CTS) towards the source, being such message overheard by any potential relay. As a result potential relays opportunistically start a self-electing procedure. Based on the information sent by self-elected relays to the destination, the later chooses the best relay or set of relays among self-elected relays (cooperative relay scheduling).

In more detail, after receiving a Request To Send (RTS) from source, if the direct link between source and destination is poor, the destination piggybacks the source-destination datarate $\left(R_{s d}\right)$ within a CTS frame. The inclusion of $R_{s d}$ is an implicit indication of the relaying initiation. With the reception of such cooperative CTS, the source sends the data frame to destination, while the potential relays start opportunistic relay selection process after overhearing the cooperative CTS frame (Section III-A1). After reception of the data frame from source, the destination does not send an ACK frame immediately to the source. Rather, it waits for a predefined time frame (reception windows) to allow potential relays to transmit a Qualification Message (QM) to the destination (Section III-A2). After the expiration of the reception window, the destination sends an ACK frame to the source piggybacking the ID of the selected relay or set of relays, as well as information about the relay-destination data-rate $\left(R_{r d}\right)$; the 


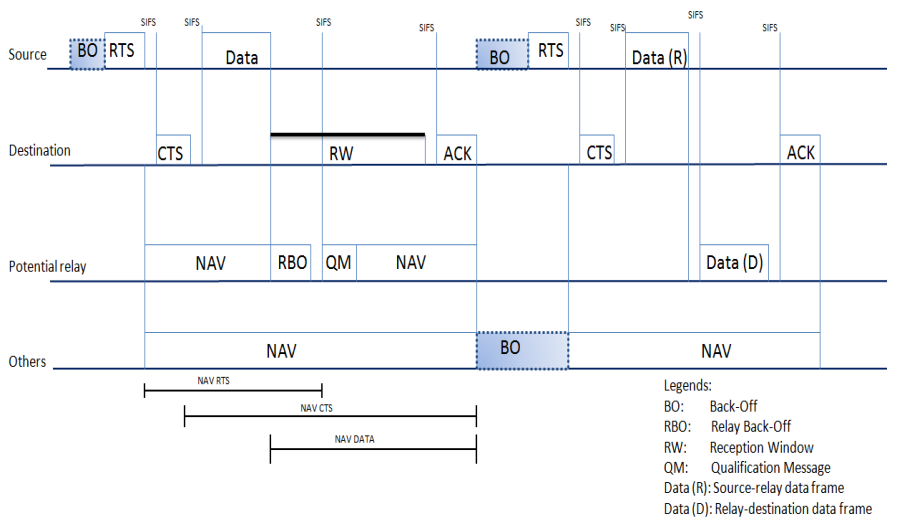

Figure 2. Proactive mode.

source can infer about the source-relay data-rate $\left(R_{s r}\right)$ by overhearing the QM sent to destination by potential relays.

The source then starts the cooperative transmission, in which it relays the following data frames via that selected relay with data-rates $R_{s r}$ and $R_{r d}$. This procedure continues until the quality of the direct link improves and, as a consequence, the destination send an ACK without relay ID and $R_{r d}$.

1) Opportunistic Relay Selection: In case of need for relaying, relays start by verifying if they are inside the cooperation area by computing their Cooperation Factor $(\mathrm{CF})$ as given in Equation 1. These rates are computed by overhearing RTS and CTS frames exchanged between source and destination. The CF ensures that potential relays are closely bounded with the source while having good channel towards the destination: an eligible relay must have a $\mathrm{CF}$ that ensures a higher $R_{s r}$ and $R_{r d}$ data-rate than over the direct link from source to destination.

$$
C F=\left(R_{s r} * R_{r d}\right) /\left(R_{s r}+R_{r d}\right), C F \in[0, \infty[
$$

The relay self-election procedure relies upon node degree and traffic load to compute the overall interference level (I) that each node is subjected to [7]. If self-elected to operate as a relay, a node computes its contention window [7]. The contention window plays an important role in scheduling relay opportunities. The goal is to increase the probability of successful transmissions from relays to the destination by giving more priority to relays that are more closely bounded to the destination, and have less interference. Upon expiration of the contention window, relays try to send a QM to the destination, showing its qualification as a relay.

2) Cooperative Relay Scheduling: After reception of qualification messages from all self-elected relays, the destination estimates which of the involved relays are more suitable to help in subsequent transmissions from that source. To get multiple qualification messages the destination only processes the received qualification messages after a predefined time window, i.e., Reception Window (RW). The size of the reception windows is of major importance, since it will have an impact upon the number of qualification messages that will be considered by the destination (this issue is further explained in section IV).

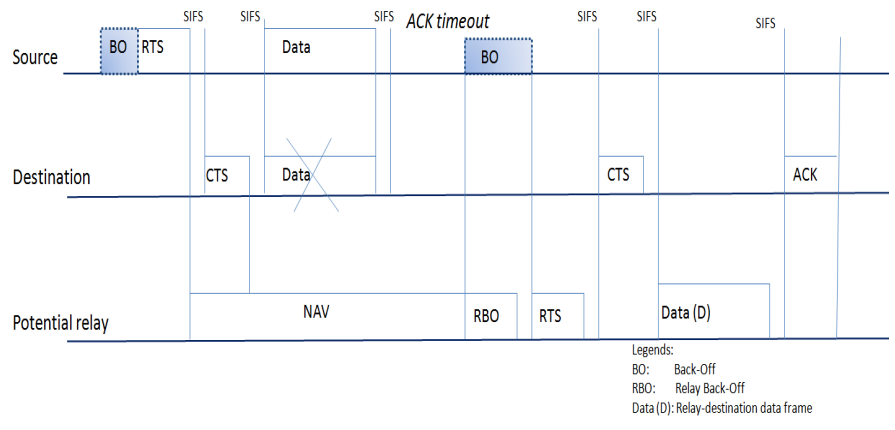

Figure 3. Reactive mode.

After the expiration of the reception window the destination processes all the received qualification messages based upon their received signal strength $\left(R_{r d}\right)$ and $R_{s r}$, which is carried by the QM. The destination sends an ACK frame to the source including the ID (i.e., MAC address) of the selected relay, which can continue sending received frames to the destination. If no QM is received, the destination sends a normal ACK to the source (without indication of a relay).

Although it is true that cooperative relay scheduling introduces a delay while selecting a relay, that operation occurs only during the relay selection phase. During cooperative transmission phase, data frames are relayed via selected relay without contention and without further delays.

\section{B. Reactive Operation}

RelaySpot allows the relays to forward data frames on behalf of the source if the direct link between source and destination fails.

If an overhearing node detects a failed direct transmission (missing ACK) due to collisions or interference, it tries to send the overheard data frame on behalf of source to avoid retransmission. In this case, first the self-election process takes place (Section III-A1), ending with the self-elected relays scheduling their $\mathrm{CW}$ for transmission to the destination. The relay whose $\mathrm{CW}$ expires first, forwards the overheard data frame. In contrast to the proactive mode, in the reactive mode the qualified relay does not send a QM; rather the relay sends the data frame directly to the destination. Therefore, in this case no scheduler is used at the destination. Upon overhearing the relay-destination transmission, the source stops the retransmission process. As we are not using any QM or scheduler, the potential relay that gets the channel first retransmits the failed data. If the source get the channel first, it will retransmit and in this case the relays drop the frame. An example is provided in Figure 3, where the destination did not receive the data frame from the source.

1) Relay Switching: While the operation described in Section III-B aims to allow RelaySpot to react to a failure of the direct link, the relay switching functionality aims to compensate for unsuccessful relay transmissions. Relay selection faces several optimization problems that are difficult to solve, which means that the best relay may be difficult to find by the destination based on the set of potential relays. Hence, aiming to be suitable for dynamic scenarios, RelaySpot allows 
the destination to select the best possible relay. In order to keep a good quality level in case of a bad decision from the destination, RelaySpot allows potential relays to cooperate for replacing the current one, by asking the source to switch the relay for the subsequent data frames.

If a potential relay is not selected in the relay selection procedure, but it can provide better performance, it sends a Switching Message (SM) to the destination. This way the previously selected relay can be switched to the newly selected relay, since: i) by overhearing the SM frame sent by the new relay, the source will send the next data frame towards the new relay: ii) by receiving the SM frame sent by the new relay, the destination knows that the next data frame will be sent by it.

Relay switching is very suitable for dynamic scenarios where a previously selected relay may not be efficient at some stage, for instance due to mobility, fading, or obstacles. Hence, unlike prior-art, relay switching can overcome such variations in network conditions making the deployment of cooperative relaying possible for dynamic networks.

Relay switching is also used to keep data being relayed in the presence of a failed relay. If a potential relay detects that the cooperative transmission via a relay failed, it tries to retransmit the failed data frame (according to III-B), implicitly leading to relay switching.

\section{PERformance ANALYSiS}

This section analyzes the RelaySpot hybrid protocol, by providing some preparatory analysis, and comparisons with two proactive benchmarks: opportunistic and broadcast-based approaches.

\section{A. Benchmarks}

As mentioned before, it is still not clear if hybrid approaches are better than proactive opportunistic and broadcast-based approaches in terms of improving network capacity. For this purpose we implement a generic proactive opportunistic and broadcast-based relaying, based on the analysis made of prior art, as described in section 2 .

In opportunistic relaying, the source sends data frames at fast bit-rate (i.e., $11 \mathrm{Mbps}$ ), while reserving channel for two fast bit-rate transmissions (source-relay and relay-destination transmissions). The overhearing nodes try to forward the data frame to the destination after performing a back-off for 300 us [12]. This is a purely opportunistic behavior, as the source does not know the availability of relays. This process is repeated for every frame that needs to be relayed.

To implement a generic broadcast-based relaying approach, we consider a proactive source-based mechanism. We assume passive overhearing, based on which the source updates a cooperative table and selects a relay prior to transmission. To initiate a transmission via a relay the source includes the relay address within address 4 of RTS frame [8]. The relay responds by sending a control frame of type CTS. After the source receives a CTS from destination, it starts a cooperative transmission similar to RelaySpot.
Table I

SIMULATION PARAMETERS

\begin{tabular}{|l|l|}
\hline Parameter & Values \\
\hline Playground Size & $200 \times 200 \mathrm{~m}^{2}$ \\
\hline Path Loss Coefficient & 4 \\
\hline Carrier Frequency & $2.412 \mathrm{e} 9 \mathrm{~Hz}$ \\
\hline Max Transmission Power & $100 \mathrm{~mW}$ \\
\hline Signal Attenuation Threshold & $-120 \mathrm{dBm}$ \\
\hline MAC Header Length & $272 \mathrm{bits}$ \\
\hline MAC Queue Length & 14 frames \\
\hline Basic Bitrate & $1 \mathrm{Mbps}$ \\
\hline Rts-Cts Threshold & $400 \mathrm{bytes}$ \\
\hline Thermal Noise & $-110 \mathrm{dBm}$ \\
\hline MAC Neighborhood Max Age & $100 \mathrm{~s}$ \\
\hline Payload Size & $1 \mathrm{~K}$ bytes \\
\hline
\end{tabular}

\section{B. Simulation Setup}

Evaluation is based on simulations run on the MiXiM framework of the OMNeT++ 4.1 simulator. Table I lists the simulation parameters. Each simulation has a duration of 300 seconds and is run ten different times in order to provide results with a $95 \%$ confidence interval. Simulations consider a scenario where all nodes are static.

This paper investigates a typical WLAN composed of one AP and 25 nodes distributed randomly (each node is a source and a potential relay). Each node is equipped with only one half-duplex transceiver and allocated a unique MAC address. All the nodes in the network transmit control frames and data frames with the same power. One wireless channel is shared by all nodes and all frames are transmitted over it. The network can support four different rates $(1,2,5.5$ and $11 \mathrm{Mbps}$ ) determined by the distance of the node to the AP, while the control frames are transmitted at basic rate.

\section{Preparatory Analysis}

The impact of reception window in the performance of hybrid approaches can be significant. Therefore, we perform simulations using relay selection with scheduler, at network load of $10 \mathrm{~K}$ frames per second. In what concerns the size of the reception window, results (c.f. Figure 4) show that it is better to have a big reception window in order to allow the AP to grab a larger number of QMs, allowing it to select the best relay with high probability.

The QM has a size of 112 bits, transmitted at the basic bitrate, which means that the transmission of QM takes 304 us. A very small reception window (604 us in figure) allows the AP to receive only one QM, which means that the destination has only one relay to select from. Such relay is with high probability a node closer to the source, since such nodes overhear good copies of source frames first. Moreover, in case of collision of QMs, the destination is not able to select a relay, leading to low throughput especially with higher node density. Our findings show that a reception window of size 1504 us provides an overall average throughput gain of $44 \%$ in relation to the direct link. Contrary to what could be expected, our findings show that throughput gain increases with a large reception window. Although the reception window introduces a delay in the response of the destination, this only occurs during relay selection and not during the process of data 


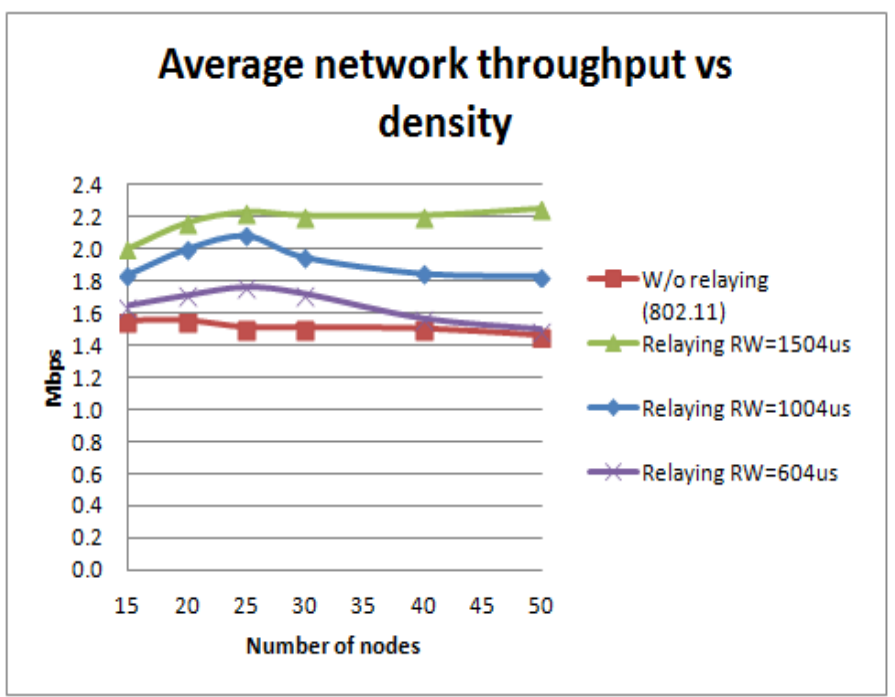

Figure 4. Analysis of impact of reception window.

relaying. Therefore, for rest of the experiments, we set the reception window to 1504 us.

\section{Impact of Interference}

This set of simulations aim to study the impact of the cooperative relay scheduling in a scenario where relays are subjected to interference (to be notice that all prior art considered scenarios without interference). Figure 5 shows that in the presence of interference, hybrid approaches, such as RelaySpot, have better performance than IEEE 802.11: RelaySpot allows higher number of transmission opportunities, avoids selecting overloaded nodes as relays, and select relays with low blockage probabilities. In this set of simulations, we consider a scenario where one source is placed at a distance from AP to observe poor data-rate, and interference is added by randomly placing transmission pairs (each with $5 \mathrm{Mbps}$ in average) among the available 25 nodes. Results show that with the introduction of interference the gain in throughput and latency drops linearly as the probability for the source to get the channel decreases. However, RelaySpot achieves a high throughput (147\% higher in average in relation to 802.11), due to its capability to select relays with better transmission success rate towards the destination. Since those relays are within the cooperation area, the condition supported by Equation 1 (i.e., $\mathrm{CF}>\mathrm{Rsd}$ ).

In term of latency RelaySpot gain is of $148 \%$ in average in relation to 802.11 , since RelaySpot is able to select relays with low load of concurrent neighbor flows, leading to a higher number of successful transmission opportunities for the relay, as the relay faces low blockage which lower the latency.

Figure 5 shows that the gain in both throughput and latency stabilize at an interference level of $25 \mathrm{Mbps}$, which is a better result than using RelaySpot without scheduler [7], [6], where the gain only stabilizes at an interference level of 60 Mbps. The reason is that the relay selection mechanism is able to schedule relays with less blockage probability, while the scheduler at the destination is able to chose a relay with better

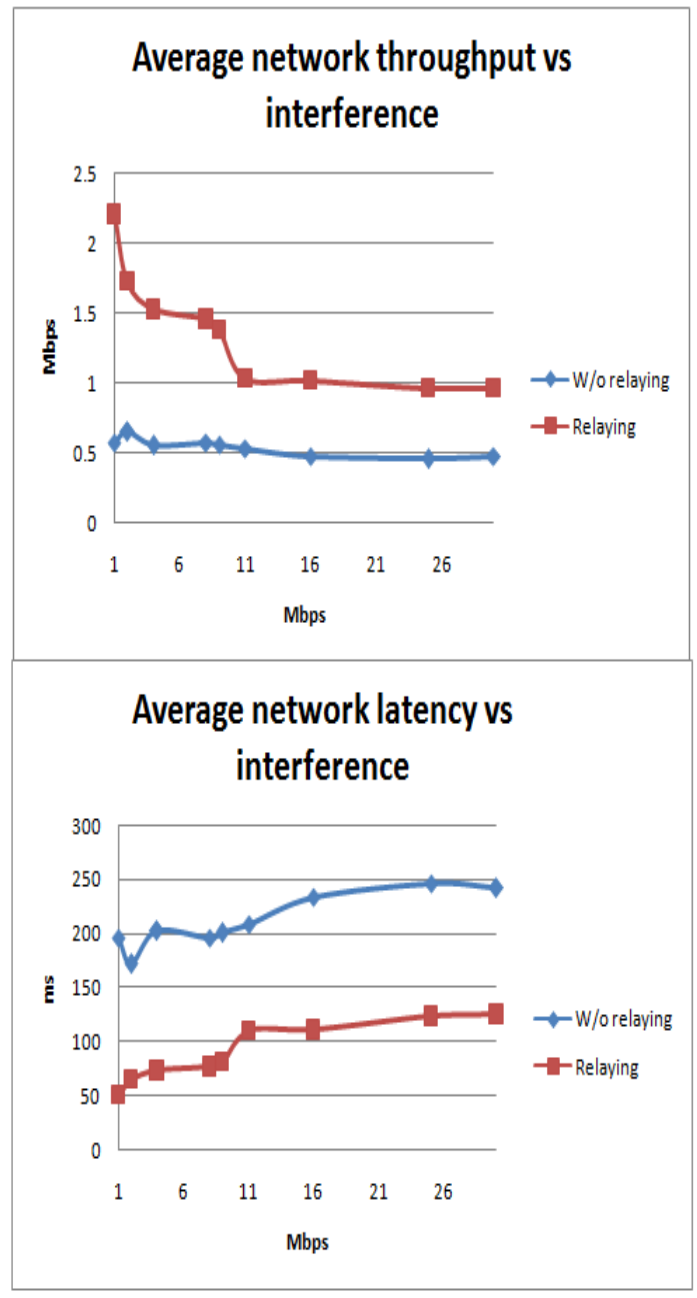

Figure 5. Analysis of impact of interference.

rate, among the qualified relays, ensuring a good performance with high interference.

\section{E. Comparisons}

In this set of simulations we compare the hybrid approach, RelaySpot, including scheduler and switching functionality, with a generic implementation of opportunistic and broadcastbased approaches. The simulation set up is the same as in Section IV-C. Figure 6 shows a clear advantage of using a hybrid approach able to react to relay failures by exploring a relay switching functionality. Due to relay switching the throughput gain is of $63 \%$ as compared to 802.11, which is an increase of $19 \%$ when compared with the operation of RelaySpot without switching (as given in Section IV-C).

Although the usage of multiple relays has its own overhead [15], in case of relay switching only one relay is selected at each time while other relays can cooperate at time when needed. Moreover, the choice of relays to react is not limited to only specific predefined set of relays. Switching can decrease the overall contention by avoiding relay re-selection and replacing relays by the better opportunistic node.

In order to analyze how much can we contribute to a good network capacity by organizing relays on-the-fly (scheduling 


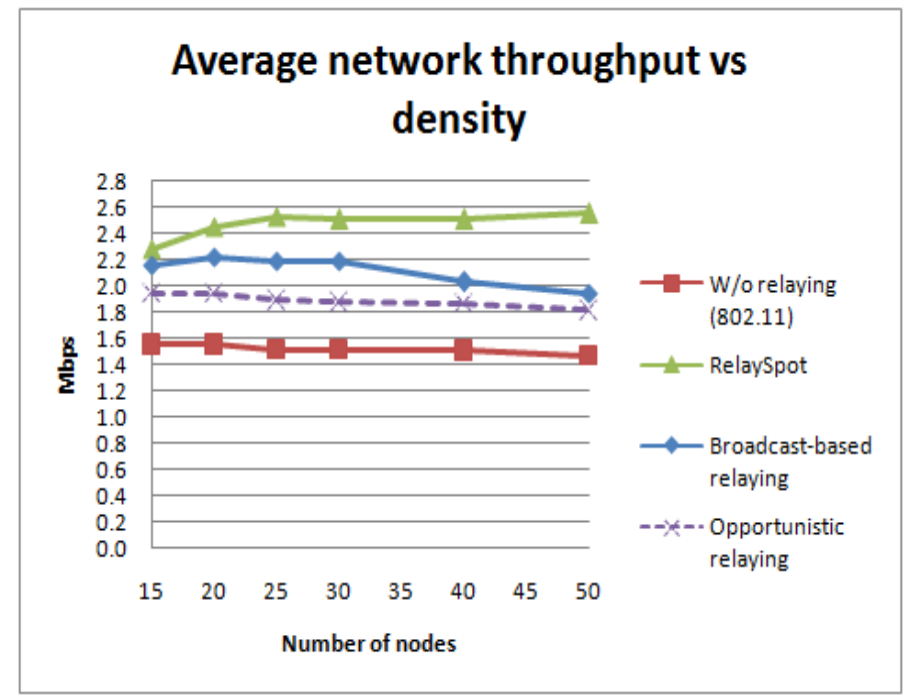

Figure 6. RelaySpot Analysis.

and switching), we compare the proposed hybrid approach with proactive broadcast-based and opportunistic relaying.

The broadcast-based relaying includes additional control messages for handshake to avoid collisions and to guaranty correct channel reservations. This is why it achieves a throughput gain of $40 \%$ in average in relation to IEEE 802.11. However, the gain decreases with the increase of network density. The reason is that relay failure increases due to collisions.

Both types of approaches (RelaySpot and broadcast-based) achieve better gain as compared to opportunistic-based relaying. Figure 6 shows that opportunistic relaying achieves a throughput gain of $24 \%$ in average in relation to IEEE 802.11. Since, the source or destination does not know the availability of relays, there is a high probability of failed relay attempt, and of collision, which limits the performance gain. Therefore, we conclude that hybrid approaches (e.g., RelaySpot) are a good cooperative relaying approach to increase the overall network performance, while decreasing the impact of relaying overhead.

\section{CONCLUSIONS}

In this paper, a hybrid relaying protocol, called RelaySpot is analyzed. This protocol aims to improve the performance of wireless networks by an efficient combination of proactive (broadcast-based and opportunistic) and reactive relaying. With RelaySpot, a relay is chosen for a cooperative transmission opportunistically, without any broadcast overhead. The relay is selected cooperatively without maintaining any table. The cooperative transmission takes place without any further contention or handshake messages. Poor relay selection and relay failures are adjusted dynamically without expecting the relay selection procedure. Therefore, we conclude that such hybrid behavior has potential to rectify drawbacks that occur in prior art, in what concern broadcast-based and opportunistic relaying.

From an experimental prospective, simulation results show that the proposed hybrid relaying achieves a throughput gain of $32 \%$ and $18 \%$ in average in relation to proactive opportunistic and broadcast-based relaying respectively (at the network load of $10 \mathrm{~K}$ frames per second, under varying network density). Moreover, hybrid relaying can effectively increase the transmission capacity of wireless local area networks, even in the presence of interference. This is the reason why RelaySpot achieves higher gain as compared to RelaySpot without scheduling mechanism [7], [6].

As future work we aim to investigate synchronization of simultaneous cooperative relays in scenarios with wireless diversity higher than one, which is possible with RelaySpot by having the destination selecting more than one relay.

\section{ACKNOWLEDGMENT}

Thanks are due to ULOOP project (257418) and FCT for $\mathrm{PhD}$ grant (SFRH/BD/60436/2009).

\section{REFERENCES}

[1] A. Ozgur, O. Leveque, and D. Tse, "Hierarchical Cooperation Achieves Optimal Capacity Scaling in Ad Hoc Networks," IEEE Transactions on Information Theory, vol. 53, no. 10, pp. 3549-3572, 2007.

[2] W. Elmenreich, N. Marchenko, H. Adam, C. Hofbauer, G. Brandner, C. Bettstetter, and M. Huemer, "Building Blocks of Cooperative Relaying in Wireless Systems," Electrical and Computer Engineering, Springer, vol. 125, no. 10, pp. 353-359, Oct. 2008.

[3] T. Jamal, P. Mendes, and A. Zúquete, "Wireless Cooperative Relaying Based on Opportunistic Relay Selection," International Journal on Advances in Networks and Services, vol. 05, no. 2, pp. 116-127, Jun. 2012.

[4] T. Jamal and P. Mendes, "Relay Selection Approaches for Wireless Cooperative Networks," in Proc. of IEEE WiMob, Niagara Falls, Canada, Oct. 2010.

[5] T. Jamal, P. Mendes, and A. Zúquete, "RelaySpot: A Framework for Opportunistic Cooperative Relaying," in Proc. of IARIA ACCESS, Luxembourg, Jun. 2011.

[6] T. Jamal, P. Mendes, and A. Zúquete, "Interference-Aware Opportunistic Relay Selection," in Proc. of ACM CoNEXT student workshop, Tokyo, Japan, Dec. 2011.

[7] T. Jamal, P. Mendes, and A. Zúquete, "Opportunistic Relay Selection for Wireless Cooperative Network," in Proc. of IEEE IFIP NTMS, Istanbul, Turkey, May 2012.

[8] P. Liu, Z. Tao, S. Narayanan, T. Korakis, and S. Panwar, "CoopMAC: A Cooperative MAC for Wireless LANs," IEEE Journal on Selected Areas in Communications, vol. 25, no. 2, pp. 340-354, Feb. 2007.

[9] K. Tan, Z. Wan, H. Zhu, and J. Andrian, "CODE: Cooperative Medium Access for Multirate Wireless Ad Hoc Network," in Proc. IEEE of SECON, California, USA, Jun. 2007.

[10] H. Zhu and G. Cao, "rDCF: A Relay-Enabled Medium Access Control Protocol for Wireless Ad Hoc Networks," IEEE Transactions on Mobile Computing, vol. 5, Sep. 2006.

[11] G. Bocherer and R. Mathar, "On the Throughput/Bit-Cost Tradeoff in CSMA Based Cooperative Networks," in Proc. of ITG Conference on Source and Channel Coding (SCC), 2010.

[12] L. M. Feeney, B. Cetin, D. Hollos, M. Kubisch, S. Mengesha, and H. Kar, "Multi-rate Relaying for Performance Improvement in IEEE 802.11 WLANs," in Proc. of WWIC, Coimbra, Portugal, May 2007.

[13] L. Mei-Hsuan, S. Peter, and C. Tsuhan, "Design, Implementation and Evaluation of an Efficient Opportunistic Retransmission Protocol," in Proc. of IEEE MobiCom, Beijing, China, Apr. 2009.

[14] S. Shankar, C. Chou, and M. Ghosh, "Cooperative Communication MAC (CMAC): a New MAC Protocol for Next Generation Wireless LANs," in Proc. of International Conference on Wireless Networks, Communications and Mobile Computing, Maui, Hawaii, 2005.

[15] C. Chou, J. Yang, and D. Wang, "Cooperative MAC Protocol with Automatic Relay Selection in Distributed Wireless Networks," in Proc. IEEE PerComW07, 2007, pp. 526 - 531. 